\title{
Multiple Lie Derivatives and Forests
}

\author{
Florent HIVERT and Nefton PALI
}

\begin{abstract}
We obtain a complete time expansion of the pull-back operator generated by a real analytic flow of real analytic automorphisms acting on analytic tensor sections of a manifold. Our expansion is given in terms of multiple Lie derivatives. Motivated by this expansion, we provide a rather simple and explicit estimate for higher order covariant derivatives of multiple Lie derivatives acting on smooth endomorphism sections of the tangent bundle of a manifold. We assume the covariant derivative to be torsion free. The estimate is given in terms of Dyck polynomials. The proof uses a new result on the combinatorics of rooted labeled ordered forests and Dyck polynomials.
\end{abstract}

\section{Notations and motivation}

In this paper the products over ordered sets of indices are always considered from the left to the right with respect to the order structure of the set. For any integer $k \geqslant 1$, we set $[k]:=\{1, \ldots, k\}$ and we denote by $\lambda \vDash k$ any element $\lambda \equiv\left(\lambda_{1}, \ldots, \lambda_{l_{\lambda}}\right) \in \mathbb{N}_{>0}^{l_{\lambda}}$ such that $\sum_{j=1}^{l_{\lambda}} \lambda_{j}=k$. We denote by $\mathbb{N}^{l}(p)$ the set of $P \equiv\left(p_{1}, \ldots, p_{l}\right) \in \mathbb{N}^{l}$ such that $\sum_{j=1}^{l} p_{j}=p$.

We start now with a general remark. Let $\left(\varphi_{t}\right)_{t \in(-\varepsilon, \varepsilon)}$ be a real analytic family of real analytic automorphisms of a real analytic manifold $X$ with $\varphi_{0}=\mathrm{id}_{X}$ and let $\xi(t):=\dot{\varphi}_{t} \circ \varphi_{t}^{-1}=\sum_{k \geqslant 0} \xi_{k} t^{k}$. The pull back operator $\varphi_{t}^{*}$ acting on real analytic sections of the tensor bundles $\left(T_{X}^{*}\right)^{\otimes p} \otimes T_{X}^{\otimes q}$, with $p, q \geqslant 0$, can be expanded as

$$
\varphi_{t}^{*}=\mathbb{I}+\sum_{k \geqslant 1} t^{k} \sum_{\lambda \vDash k} \prod_{j=1}^{l_{\lambda}} L_{|\lambda|_{j}^{-1} \xi_{\lambda_{j}-1}},
$$

where $|\lambda|_{j}:=\sum_{r=1}^{j} \lambda_{r}$. Of course we can relax the analyticity assumption to smooth on the ground variable $x \in X$, but for our future applications we will need to stay in the real analytic category.

We equip the Sobolev space $H^{r}\left(X,\left(T_{X}^{*}\right)^{\otimes q} \otimes T_{X}^{\otimes p}\right)$ with the Sobolev norm $\|\cdot\|_{r}$ obtained using the covariant derivatives and the pointwise max norm on multilinear forms with respect to a smooth Riemannian metric $g$. This norm is equivalent to the usual Sobolev norm defined by means of partitions of unity. We remind that the space $H^{r}\left(X,\left(T_{X}^{*}\right)^{\otimes q} \otimes T_{X}^{\otimes p}\right)$ is an algebra for $r \in \mathbb{N}$ sufficiently 
big and for such $r$ hold the inequality $\|u v\|_{r} \leqslant C_{r}\|u\|_{r}\|v\|_{r}$, for some constant $C_{r}>0$. From now on we fix such an $r$. Sobolev norms are quite natural for Hardy spaces. In any case the estimate in the main theorem below hold with respect to any algebra norm.

A case of major interest is when the pull back operator act on analytic endomorphism sections of the tangent bundle. Indeed this is the case when we consider a complex structure $J$ over a complex manifold $X$ and we wish to study the dynamics of the flow $\varphi_{t}^{*} J$. As explained in [P-S, Pal2, Pal3], among others, this is a central problem in complex differential geometry related with a strong version of the Hamilton-Tian conjecture (See [Pal3]). For the applications it is very important to have an explicit and simple estimate of the multiple Lie derivatives that appear in the expansion (1.1) and their higher order covariant derivatives. This is provided by the following result, which is our main theorem.

Theorem 1. (MainTheorem) Let $\nabla$ be the extension to tensor sections of any torsion free connection and let $A$ be a smooth endomorphism section of the tangent bundle. Then for any family of smooth vector fields $\left(\xi_{j}\right)_{j=1}^{k}$ the estimate holds

$$
\begin{gathered}
\frac{1}{h !}\left\|\nabla^{h}\left(\prod_{j=1}^{k} L_{\xi_{j}}\right) A\right\|_{r} \\
\leqslant C_{r}^{k} \sum_{\substack{P \in \operatorname{Dyck}(k) \\
H \in \mathbb{N}^{k+1}(h)}} C_{P} \frac{1}{h_{k+1} !}\left\|\nabla^{h_{k+1}+D_{P, k}} A\right\|_{r} \prod_{j=1}^{k} \frac{1}{h_{j} !}\left\|\nabla^{h_{j}+p_{j}} \xi_{j}\right\|_{r},
\end{gathered}
$$

where

$$
\begin{aligned}
& \operatorname{Dyck}(k):=\left\{P \equiv\left(p_{1}, \ldots, p_{k}\right) \in \mathbb{N}^{k} \mid \sum_{1 \leqslant r \leqslant j} p_{r} \leqslant j, \forall j \in[k]\right\}, \\
& D_{P, j}:=j-\sum_{1 \leqslant r \leqslant j} p_{r}, \\
& C_{P}:=\prod_{j=1}^{k}\left[2\left(\begin{array}{c}
D_{P, j-1} \\
p_{j}-1
\end{array}\right)+\left(\begin{array}{c}
D_{P, j-1} \\
p_{j}
\end{array}\right)\right] \\
&=\prod_{\substack{1 \leqslant j \leqslant k \\
p_{j} \neq 0}}\left(2+\frac{D_{P, j}}{p_{j}}\right)\left(\begin{array}{c}
D_{P, j-1} \\
p_{j}-1
\end{array}\right),
\end{aligned}
$$

with the convention that $\left(\begin{array}{c}m \\ n\end{array}\right)=0$ in $n \notin\{0,1, \ldots, m\}$.

The elements $P$ of $\operatorname{Dyck}(k)$ are called Dyck vectors of length $k$. Each Dyck vector is associated to a Dyck monomial $X^{P}=X_{1}^{p_{1}} \cdots X_{k}^{p_{k}}$. We give below 
their full list for $k=1,2,3$ together with the associated $C_{P}$ coefficient

$$
\operatorname{Dyck}(1): \quad(0) 1 \quad \text { (1) } 2
$$

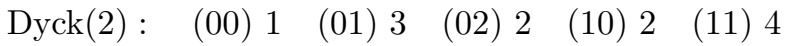

$$
\begin{aligned}
& \text { Dyck(3): } \begin{array}{lllllll}
(000) 1 & (001) 4 & (002) 5 & (003) 2 & (010) 3 & (011) 9 & (012) 6 \\
(020) 2 & (021) 4 & (100) 2 & (101) 6 & (102) 4 & (110) 4 & (111) 8
\end{array}
\end{aligned}
$$

It easy to see that the cardinality $|\operatorname{Dyck}(k)|$ is the $k+1$-th Catalan number $C_{k+1}$ where $C_{k}=\frac{1}{k+1}\left(\begin{array}{c}2 k \\ k\end{array}\right)$. Indeed $C_{k}$ is known (see OEIS] sequence A000108) to be the number of so called Dyck path, that is lattice path on the grid $\mathbb{N} \times \mathbb{N}$, starting from $(0,0)$ ending at $(k, k)$ with only North and East step and staying under the diagonal. As illustrated below, such a path can be bijectively encoded by the length of the vertical segment, omitting the last one. Requiring that the path stay below the diagonal is equivalent to the condition $\sum_{1 \leqslant r \leqslant j} p_{r} \leqslant j$

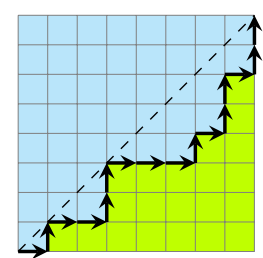

1020012

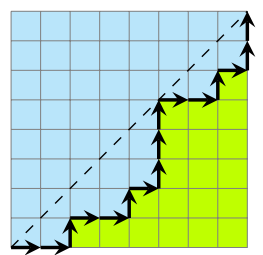

0101301

Example 1. We now illustrate the computation of $C_{P}$. Let $P=(0,1,0,1,3,0,1)$. Then the value of $D_{P, j}$ are given by the following array:

\begin{tabular}{|c|cccccccc|}
\hline$j$ & 0 & 1 & 2 & 3 & 4 & 5 & 6 & 7 \\
\hline$p_{j}$ & & 0 & 1 & 0 & 1 & 3 & 0 & 1 \\
$D_{P, j}$ & 0 & 1 & 1 & 2 & 2 & 0 & 1 & 1 \\
\hline
\end{tabular}

So that

$$
\begin{aligned}
C_{p} & =\left[2\left(\begin{array}{c}
0 \\
-1
\end{array}\right)+\left(\begin{array}{l}
0 \\
0
\end{array}\right)\right]\left[2\left(\begin{array}{l}
1 \\
0
\end{array}\right)+\left(\begin{array}{l}
1 \\
1
\end{array}\right)\right]\left[2\left(\begin{array}{c}
1 \\
-1
\end{array}\right)+\left(\begin{array}{l}
1 \\
0
\end{array}\right)\right] \times \\
& \times\left[2\left(\begin{array}{l}
2 \\
0
\end{array}\right)+\left(\begin{array}{l}
2 \\
1
\end{array}\right)\right]\left[2\left(\begin{array}{l}
2 \\
2
\end{array}\right)+\left(\begin{array}{l}
2 \\
3
\end{array}\right)\right]\left[2\left(\begin{array}{c}
0 \\
-1
\end{array}\right)+\left(\begin{array}{l}
0 \\
0
\end{array}\right)\right]\left[2\left(\begin{array}{l}
1 \\
0
\end{array}\right)+\left(\begin{array}{l}
1 \\
1
\end{array}\right)\right] \\
& =72 .
\end{aligned}
$$

\section{Proof of the expansion formula (1.1)}

We remind first (see for instance lemma 27 in the sub-section 19.4 on page 916 of [Pal1]) the well known derivation rule

$$
\frac{d}{d t}\left(\varphi_{t}^{*} \alpha_{t}\right)=\varphi_{t}^{*}\left(\frac{d}{d t} \alpha_{t}+L_{\xi_{t}} \alpha_{t}\right)
$$


for any curve $t \longmapsto \alpha_{t} \in\left(T_{X}^{*}\right)^{\otimes p} \otimes T_{X}^{\otimes q}$. What we need to prove in order to obtain (1.1) is the formula

$$
\frac{1}{k !}{\frac{d^{k}}{d t^{k}}}_{\left.\right|_{t=0}}\left(\varphi_{t}^{*} \alpha\right)=\sum_{\lambda \models k}\left[\prod_{j=1}^{l_{\lambda}} L_{|\lambda|_{j}^{-1} \xi_{\lambda_{j}-1}}\right] \alpha,
$$

with $\alpha \in\left(T_{X}^{*}\right)^{\otimes p} \otimes T_{X}^{\otimes q}$, that we rewrite under the form

$$
\begin{aligned}
\left.\frac{d^{k}}{d t^{k}}\right|_{t=0}\left(\varphi_{t}^{*} \alpha\right) & =\sum_{\lambda \models k} C_{\lambda}\left[\prod_{j=1}^{l_{\lambda}} L_{\xi_{0}}^{\left(\lambda_{j}-1\right)}\right] \alpha, \\
C_{\lambda} & =\frac{|\lambda| !}{\prod_{j=1}^{r}\left[\left(\lambda_{j}-1\right) !|\lambda|_{j}\right]}, \\
\xi_{t}^{(k)} & :=\frac{d^{k} \xi_{t}}{d t^{k}}
\end{aligned}
$$

with the convention $0 !=1$. We will prove the more general formula

$$
\frac{d^{k}}{d t^{k}} \varphi_{t}^{*}=\sum_{\lambda \models k} C_{\lambda} \varphi_{t}^{*} \prod_{j=1}^{l_{\lambda}} L_{\xi_{t}^{\left(\lambda_{j}-1\right)}},
$$

by induction. (Obviously the above formula is true for $k=1$.) Taking one more derivative we obtain thanks to (2.1)

$$
\frac{d^{k+1}}{d t^{k+1}} \varphi_{t}^{*}=\sum_{\lambda \models k} C_{\lambda} \varphi_{t}^{*}\left[\sum_{s=1}^{l_{\lambda}} \prod_{j=1}^{l_{\lambda}} L_{\xi_{t}^{\left(\lambda_{j}+\delta_{j, s}-1\right)}}+L_{\xi_{t}} \prod_{j=1}^{l_{\lambda}} L_{\xi_{t}^{\left(\lambda_{j}-1\right)}}\right] .
$$

If we identify formally the product $\varphi_{t}^{*} \prod_{j=1}^{l_{\lambda}} L_{\xi_{t}^{\left(\lambda_{j}-1\right)}}$ with the composition $\lambda$ then the previous sum corresponds to the formal sum of compositions

$$
S_{k}:=\sum_{\lambda \vDash k} C_{\lambda}\left[\lambda^{\prime}+(1, \lambda)\right]
$$

where

$$
\begin{aligned}
\lambda^{\prime} & :=\sum_{j=1}^{l_{\lambda}}\left(\lambda_{1}, \ldots, \lambda_{j}+1, \ldots, \lambda_{l_{\lambda}}\right), \\
(1, \lambda) & :=\left(1, \lambda_{1}, \ldots, \lambda_{l_{\lambda}}\right) .
\end{aligned}
$$

We observe that the operation which associates to any composition $\lambda$ the components of the formal sum $\lambda^{\prime}+(1, \lambda)$, generates all the compositions of $k+1$. For any $\lambda \vDash k$ and $\Lambda \vDash k+1$ let us write $\lambda \rightarrow \Lambda$ if $\Lambda=\left(\lambda_{1}, \ldots, \lambda_{j}+1, \ldots, \lambda_{l_{\lambda}}\right)$ for some $j$ or $\Lambda=(1, \lambda)$. Then Equation 2.2 rewrites as

$$
S_{k}=\sum_{\lambda \vDash k} C_{\lambda} \sum_{\substack{\Lambda \models k+1 \\ \lambda \rightarrow \Lambda}} \Lambda
$$


Exchanging the two sums yield

$$
S_{k}=\sum_{\Lambda \models k+1}\left(\sum_{\substack{\lambda \models k \\ \lambda \rightarrow \Lambda}} C_{\lambda}\right) \Lambda .
$$

We observe that $\Lambda \vDash k+1$ being fixed, the $\lambda \vDash k$ such that $\lambda \rightarrow \Lambda$ are obtained either by removing 1 in front of $\Lambda$ (if $\Lambda$ starts with 1 ) or by decreasing any component of $\Lambda$ greater that 2 .

The conclusion of the induction will follow from the equality $C_{\Lambda}=C_{\Lambda}^{\prime}$, which rewrites in a more explicit form as

$$
\frac{|\Lambda| !}{\prod_{j=1}^{l_{\Lambda}}\left[\left(\Lambda_{j}-1\right) !|\Lambda|_{j}\right]}=\sum_{s=1}^{l_{\Lambda}} \frac{(|\Lambda|-1) !}{\prod_{j=1}^{l_{\Lambda}}\left[\left(\Lambda_{j}-\delta_{s, j}-1\right)_{+} ! \sum_{t=1}^{j}\left(\Lambda_{t}-\delta_{s, t}\right)\right]},
$$

where $(a)_{+}:=\max \{a, 0\}$. Symplifing the common factor $(|\Lambda|-1)$ !, rearranging and setting $l:=l_{\Lambda}$ we infer that the previous equality is equivalent to

$$
\sum_{s=1}^{l} \Lambda_{s}=\sum_{s=1}^{l} \prod_{j=1}^{l} \frac{\left(\Lambda_{j}-1\right) ! \sum_{t=1}^{j} \Lambda_{t}}{\left[\left(\Lambda_{j}-\delta_{s, j}-1\right)_{+} ! \sum_{t=1}^{j}\left(\Lambda_{t}-\delta_{s, t}\right)\right]} .
$$

The later rewrites in a simpler way as

$$
\sum_{s=1}^{l} \Lambda_{s}=\sum_{s=1}^{l}\left(\Lambda_{s}-1\right) \prod_{j=s}^{l} \frac{\sum_{t=1}^{j} \Lambda_{t}}{\sum_{t=1}^{j} \Lambda_{t}-1} .
$$

We show (2.7) by induction on $l$. The equality (2.7) is obvious for $l=1$. We decompose the sum

$$
\begin{aligned}
& \sum_{s=1}^{l+1}\left(\Lambda_{s}-1\right) \prod_{j=s}^{l+1} \frac{\sum_{t=1}^{j} \Lambda_{t}}{\sum_{t=1}^{j} \Lambda_{t}-1} \\
& =\left(\Lambda_{l+1}-1\right) \frac{\sum_{t=1}^{l+1} \Lambda_{t}}{\sum_{t=1}^{l+1} \Lambda_{t}-1} \\
& \quad+\frac{\sum_{t=1}^{l+1} \Lambda_{t}}{\sum_{t=1}^{l+1} \Lambda_{t}-1} \sum_{s=1}^{l}\left(\Lambda_{s}-1\right) \prod_{j=s}^{l} \frac{\sum_{t=1}^{j} \Lambda_{t}}{\sum_{t=1}^{j} \Lambda_{t}-1} \\
& =\left(\Lambda_{l+1}-1\right) \frac{\sum_{t=1}^{l+1} \Lambda_{t}}{\sum_{t=1}^{l+1} \Lambda_{t}-1}+\frac{\sum_{t=1}^{l+1} \Lambda_{t}}{\sum_{t=1}^{l+1} \Lambda_{t}-1} \sum_{s=1}^{l} \Lambda_{s},
\end{aligned}
$$

by the inductive assumption. We conclude

$$
\begin{aligned}
\sum_{s=1}^{l+1}\left(\Lambda_{s}-1\right) \prod_{j=s}^{l+1} \frac{\sum_{t=1}^{j} \Lambda_{t}}{\sum_{t=1}^{j} \Lambda_{t}-1} & =\left(\sum_{t=1}^{l+1} \Lambda_{t}-1\right) \frac{\sum_{t=1}^{l+1} \Lambda_{t}}{\sum_{t=1}^{l+1} \Lambda_{t}-1} \\
& =\sum_{t=1}^{l+1} \Lambda_{t},
\end{aligned}
$$


which is the equality (2.7) for $l+1$.

Combinatorial proof. We give now a second more combinatorial proof of the formula giving the $C_{\lambda}$. Recall that we encoded the product

$$
\varphi_{t}^{*} \prod_{j=1}^{l_{\lambda}} L_{\xi_{t}^{\left(\lambda_{j}-1\right)}},
$$

with the composition $\lambda$. We denote $D$ the linear operator acting on formal linear combination of compositions defined by

$$
D(\lambda):=\lambda^{\prime}+(1, \lambda)=\sum_{\lambda \rightarrow \Lambda} \Lambda
$$

where we defined the relation $\rightarrow$ by $\lambda \rightarrow \Lambda$ if $\Lambda=\left(\lambda_{1}, \ldots, \lambda_{j}+1, \ldots, \lambda_{l_{\lambda}}\right)$ for some $j$ or $\Lambda=(1, \lambda)$. Then

$$
\frac{d}{d t}\left(\varphi_{t}^{*} \prod_{j=1}^{l_{\lambda}} L_{\xi_{t}^{\left(\lambda_{j}-1\right)}}\right)
$$

is encoded by $D(\lambda)$. So that to compute $\frac{d^{k}}{d t^{k}} \varphi_{t}^{*}$ we need to compute the coefficients $c_{k}$ of the expansion of $D^{k}(())=\sum_{\lambda \vDash k} c_{\lambda} \lambda$ where () denotes the empty composition of length 0 . The relation $\rightarrow$ is illustrated in Figure 1, together with the coefficient $c_{\lambda}$.

By definition of $D$ the coefficient $c_{\Lambda}$ of each node $\Lambda$ is the sum of the coefficients of its antecedents by the relation $\lambda \rightarrow \Lambda$. As a consequence it is equal to the number of pathes from () to $\Lambda$, that is sequences

$$
()=\lambda^{0} \rightarrow \lambda^{1} \rightarrow \lambda^{2} \rightarrow \cdots \rightarrow \lambda^{k}=\Lambda
$$

such that the relation $\lambda^{i} \rightarrow \lambda^{i+1}$ holds for any $i$ such that $0 \leq i<k$.

The striking observation is that the number of such paths starting from () to any composition $\Lambda$ of sum $k$ is equal to the number of partitions of the set $[k]=\{1, \ldots, k\}$. Recall that a partition of a set $S$ is a set $\Pi=\left\{\Pi_{1}, \ldots, \Pi_{r}\right\}$ of non empty disjoints sets $\Pi_{i}$ whose union is $S$. The number of partition of $[k]$ is know as the $k$-th Bell numbers. The first values are

$$
1,1,2,5,15,52,203,877,4140,21147
$$

and one can check on Figure 1 that the sum of the coefficient of the composition of sum 4 is indeed 15 . So we can expect that there is a bijection between the set of paths from () to $\Lambda$ and the set of partition $\Pi$ of $k$ verifying certain constraints depending on $\Lambda$.

We now describe such a constraint. First of all, we need a unique compact way to write a partition $\Pi=\left\{\Pi_{1}, \ldots, \Pi_{r}\right\}$ of $k$. So we write the element of each $\Pi_{i}$ in the natural order, separating the $\Pi_{i}$ by vertical bars $\mid$ and sorting the 


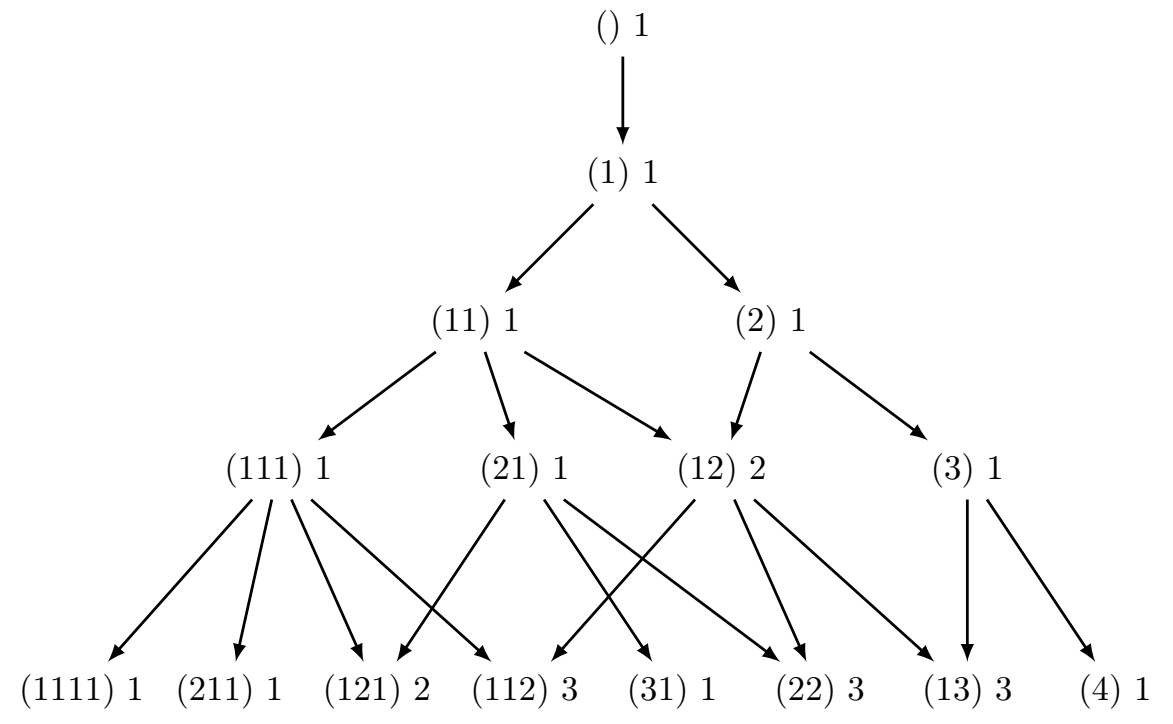

Figure 1: The relation $\lambda \rightarrow \Lambda$ and the coefficient $c_{\lambda}$

$\Pi_{i}$ among themselves according to their largest element. We call this ordering $\left(\Pi_{1}, \ldots, \Pi_{r}\right)$ the normal ordering. For example $\{\{6\},\{1\},\{7,2,4\},\{5,3\}\}$ which is a partition of $[7]$ is rather written in the order $\{\{1\},\{3,5\},\{6\},\{2,4,7\}\}$ in the compact way $1|35| 6 \mid 247$.

Definition 1. Given a partition $\Pi$ of $k$ with normal ordering $\left(\Pi_{1}, \ldots, \Pi_{r}\right)$, we call the shape of $\Pi$ and denote $\operatorname{sh}(\Pi)$ the composition $\left(\left|\Pi_{1}\right|, \ldots,\left|\Pi_{r}\right|\right)$.

For example $\operatorname{sh}(1|35| 6 \mid 247)=(1,2,1,3)$. Then we claim that

Proposition 1. For any composition $\Lambda \vDash k$, the coefficient $c_{\Lambda}$ is the number of partitions $\Pi$ such that $\operatorname{sh}(\Pi)=\Lambda$.

Before going to the proof we need some extra combinatorial ingredients. Given a partition $\Pi$ of $k>0$ we denote $\Pi^{-}$the partition of $k-1$ obtained by decreasing by 1 all the numbers in the elements of $\Pi$, removing the obtained 0 and its set as well if it is a singleton. We moreover define $\Pi^{(n)}$ by $\Pi^{(0)}=\Pi$ and $\Pi^{(n)}=\left(\Pi^{(n-1)}\right)^{-}$. For example $1|35| 6\left|247^{-}=24\right| 5 \mid 136$ and $345|26| 17^{-}=$ $234|15| 6$. Here is the sequence $1|35| 6 \mid 247^{(n)}$ for $n=0, \ldots 7$, where the second row gives the shapes:

$$
\begin{array}{cccccccc}
1|35| 6 \mid 247 & 24|5| 136 & 13|4| 25 & 2|3| 14 & 1|2| 3 & 1 \mid 2 & 1 & \emptyset \\
(1,2,1,3) & (2,1,3) & (2,1,2) & (1,1,2) & (1,1,1) & (1,1) & (1) & ()
\end{array}
$$

We notice the following obvious lemma which we just illustrated. 
Lemma 1. For all $\Pi$ partition of $k>0, \operatorname{sh}\left(\Pi^{-}\right) \rightarrow \operatorname{sh}(\Pi)$.

We now turn to the proof of Proposition 1 .

Proof. Let's denote $Q_{\Lambda}:=\{\Pi \mid \operatorname{sh}(\Pi)=\Lambda\}$ and $P_{\Lambda}$ the set of paths

$$
()=\lambda^{0} \rightarrow \lambda^{1} \rightarrow \lambda^{2} \rightarrow \cdots \rightarrow \lambda^{k}=\Lambda
$$

from () to $\Lambda$. To prove than $\left|Q_{\Lambda}\right|=\left|P_{\Lambda}\right|$ we define a bijection $F: Q_{\Lambda} \rightarrow P_{\Lambda}$ by

$$
F(\Pi)=\left(\operatorname{sh}\left(\Pi^{(k)}\right), \operatorname{sh}\left(\Pi^{(k-1)}\right), \ldots, \operatorname{sh}\left(\Pi^{(1)}\right), \operatorname{sh}\left(\Pi^{(0)}\right)\right) .
$$

Observe that $F(\Pi)$ is obtained by appending $\operatorname{sh}(\Pi)$ to $F\left(\Pi^{-}\right)$. Thanks to Lemma 1 this prove that $F(\Pi) \in P_{\Lambda}$. We now need to show that $F$ is a bijection, that is, given a path

$$
p=\left(()=\lambda^{0} \rightarrow \lambda^{1} \rightarrow \lambda^{2} \rightarrow \cdots \rightarrow \lambda^{k}=\Lambda\right),
$$

we need to show that there is a unique partition $\Theta$ such that $F(\Theta)=p$. We proceed by induction on $k$. First, for $k=0$, we observe that there is only one partition of shape (), namely the empty partition $\emptyset$. Now suppose that $\Pi=\left(\Pi_{1}, \ldots, \Pi_{r}\right)$ is the unique partition such that $F(\Pi)=\left(\lambda^{0}, \ldots, \lambda^{k}\right)$. We only need to show that there is a unique partition $\Theta$ such that $\Theta^{-}=\Pi$ and $\operatorname{sh}(\Theta)=\lambda^{k+1}$. Recall that if $\lambda^{k} \rightarrow \lambda^{k+1}$, they are two possibilities:

- Either $\lambda^{k+1}=\left(1, \lambda^{k}\right)$, in this case, the only possible $\Theta$ is

$$
\Theta=\left(\{1\}, \Pi_{1}+1, \ldots, \Pi_{r}+1\right),
$$

where for any set $S$ of integers, $S+1:=\{i+1 \mid i \in S\}$.

- Or writing $\lambda^{k}=\left(\lambda_{1}^{k}, \ldots, \lambda_{l}^{k}\right)$ there exists $j \leq l$ such that

$$
\lambda^{k+1}=\left(\lambda_{1}^{k}, \ldots, \lambda_{j}^{k}+1, \ldots, \lambda_{l}^{k}\right) .
$$

Since $\lambda^{k}=\operatorname{sh}(\Pi)$, it makes sense to define

$$
\Theta=\left(\Pi_{1}+1, \ldots,\{1\} \cup\left(\Pi_{j}+1\right), \ldots \Pi_{r}+1\right) .
$$

and again it is the only possibility.

This conclude the proof by induction on $k$.

To finish the combinatorial proof of Formula (1.1), we still need to prove the following:

Proposition 2. For any composition $\lambda \vDash k$, the number of partition $\Pi$ of $[k]$ of shape $\lambda$ is given by

$$
c_{\lambda}=\frac{|\lambda| !}{\prod_{j=1}^{r}\left[\left(\lambda_{j}-1\right) !|\lambda|_{j}\right]} .
$$


Proof. We proceed by induction on the length $r$ of any composition $\lambda$ of any sum $k$. If $r=0$, then $\lambda=()$, and the denominator product is empty so that $c_{\lambda}=1$ which is correct since the only partition is the empty one. Now to choose a partition $\Pi=\left\{\Pi_{1}, \ldots, \Pi_{r+1}\right\}$ of shape $\lambda=\left(\lambda_{1}, \ldots, \lambda_{r+1}\right)$ of $k$, we first need to choose the elements which belongs to $\Pi_{r+1}$ (which must contains at least $k$ due to the normal ordering). To get the correct shape, there must be $\lambda_{r+1}-1$ elements different from $k$ in $\Pi_{r+1}$ that must be chosen in $[k-1]=[|\lambda|-1]$. So the number of such choices is

$$
\begin{aligned}
\left(\begin{array}{c}
|\lambda|-1 \\
\lambda_{r+1}-1
\end{array}\right) & =\frac{(|\lambda|-1) !}{\left(\lambda_{r+1}-1\right) !\left(\lambda_{1}+\cdots+\lambda_{r}\right) !} \\
& =\frac{|\lambda| !}{\left(\lambda_{r+1}-1\right) !|\lambda|_{r+1}|\lambda|_{r} !},
\end{aligned}
$$

since $|\lambda|=|\lambda|_{r+1}$. We need now to choose a partition $\Theta$ of shape $\mu:=$ $\left(\lambda_{1}, \ldots, \lambda_{r}\right)$ of the remaining numbers. By naturally renumbering them, there are as many choices for $\Theta$ as partitions of $\left[|\lambda|_{r}\right]$ of shape $\left(\lambda_{1}, \ldots, \lambda_{r}\right)=\mu$. By induction they are $c_{\mu}$ of them. We therefore obtain

$$
\frac{|\lambda| !}{\left(\lambda_{r+1}-1\right) !|\lambda|_{r+1}|\lambda|_{r} !} \frac{|\lambda|_{r} !}{\prod_{j=1}^{r}\left[\left(\lambda_{j}-1\right) !|\lambda|_{j}\right]},
$$

which simplifies to the announced result.

Remark 1. The shape map sh and the coefficients $c_{\lambda}$ have a nice algebraic interpretation in terms of combinatorial Hopf algebras [HNT2]. Indeed set partition index the monomial basis $\left(\mathbf{M}_{\Pi}\right)$ of the algebra $\mathbf{W S y m}$ of symmetric function in non-commutative variables. Then the operation $\Pi \mapsto \Pi^{-}$, is encoded in the non-commutative product of WSym as

$$
\mathbf{M}_{\{\{1\}\}} \mathbf{M}_{\Pi}=\sum_{\Theta \mid \Pi=\Theta^{-}} \mathbf{M}_{\Theta} .
$$

Then [HNT2, Section 3.7] consider a quotient of WSym by the so-called stalactic congruence. To match our setting we need the right sided stalactic congruence defined by

$$
a w a \equiv w a a
$$

for all $a \in A$ and $w \in A^{*}$. This quotient amount to identify $\mathbf{M}_{\Pi}$ and $\mathbf{M}_{\Theta}$ if and only if $\operatorname{sh}(\Pi)=\operatorname{sh}(\Theta)$ leading naturally to a base $\left(N_{\lambda}\right)$ of the quotient. As a consequence our $c_{\lambda}$ are nothing but the coefficients of the expansion

$$
\frac{1}{1-N_{(1)}}=\sum_{\lambda} c_{\lambda} N_{\lambda} .
$$




\section{Multiple covariant derivatives and trees}

Let $X$ be a smooth manifold and let $\nabla$ be a covariant derivative operator acting on the smooth sections of the tangent bundle $T_{X}$. We will still denote by $\nabla$ its natural extension over tensors. For any subset $S \subset \mathbb{N}_{>0}$ we consider a family of vector fields $\left(\xi_{p}\right)_{p \in S}$ and a smooth section $A$ of the tensor bundle $\left(T_{X}^{*}\right)^{\otimes q} \otimes T_{X}^{\otimes p}$.

It is known since Cayley Cay that trees are the right tool to manipulate nested iterated derivative (see also [Man, HNT1]). He actually invented the very notion of tree for that exact purpose. In this paper we will have to deal with expression such as

$$
\nabla_{\nabla_{\xi_{2}}^{1} \xi_{3} \otimes \xi_{5} \otimes \nabla_{\xi_{1} \otimes \xi_{4}}^{2} \xi_{6}}^{3} A \equiv\left(\left(\xi_{2} \neg \nabla^{1} \xi_{3}\right) \otimes \xi_{5} \otimes\left(\left(\xi_{1} \otimes \xi_{4}\right) \neg \nabla^{2} \xi_{6}\right)\right) \neg \nabla^{3} A .
$$

We will manipulate them using trees. For example the previous expression is much easier to read if written as in the left of Figure 2. Moreover, since there is a lot of redundant information such as the $\nabla^{i}$ and the $\xi$, we will reduce it to the right of Figure 2. We remark that contrary to nature we picture trees growing from top to bottom.
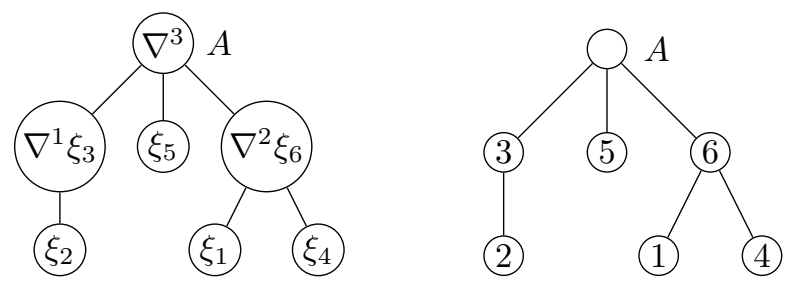

Figure 2: A nested derivative and its corresponding tree

We now define formally the kinds of tree we need in this paper. We remark that as depicted in Figure 2, they are no repeated labels in our trees so that we don't have to distinguish between a node and its label. If we orient the edge bottom-up, such a tree is just a graph of a partial function which is loopless. Moreover, for reasons that will become apparent latter, our trees have some order requirement which ensure the loopless property.

Definition 2. Let $S$ be a finite totally ordered set. A (rooted, labeled) strictly decreasing forest $F$ on $S$ is a partially defined function $F: S \rightarrow S$ such that for any $\nu$ where $F$ is defined $F(\nu)>\nu$ holds.

Elements of $S$ are called nodes of $F$. Nodes where $F$ is not defined are called roots of $F$. A forest $T$ with only one root is called a tree, in this case we denote the $\operatorname{root} \rho(T)$.

The node $F(\nu)$ is called the father of $\nu$. The preimages of $\mu \in F^{-1}(\nu)$ by $F$ are called the children of $\nu$ and we denote their set $\operatorname{Child}_{F}(\nu)$ or even Child $(\nu)$ if $F$ is clear from the context and their number by $\ell_{F}(\nu)$ or $\ell(\nu)$. Finally, When 
depicting a tree, we always draw the children in increasing order from left to right.

Note that the condition ensure that a non-empty forest must have at least one root, namely $\max S$.

We now fix some more terminology and notations: we often write the forest $F$ for its underlying set $S$ such as in $\nu \in F$ meaning $\nu \in S$. For a tree $T$, we also write $\nu \in T^{*}$ for $\nu \in T \backslash\{\rho(T)\}$.

We say that $\nu$ is a brother of $\mu$, if they have the same father. Also $\nu$ is a descendant (resp. a strict descendant) of $\mu$ if there is a $i \geq 0$ (resp. $i>0$ ) such that $\mu=F^{i}(\nu)$. Given $\mu \in S$, the set of descendant of $\mu$ determines a natural tree called the subtree of $F$ rooted at $\mu$ and denoted $F_{\mu}$. A tree $T$ of a forest $F$ is a subtree whose root $\rho(T)$ is also a root of the forest $F$. We denote their set with $\operatorname{Tree}(F)$. Of course there are as many trees in a forest as roots, their number is denoted by $l_{F}$. We denote $F^{\dagger}$ the forest obtained from $F$ by removing the tree rooted at its maximal element.

In this paper, most of the forest and trees will have their set of nodes contained in $\mathbb{N}_{>0} \cup\{0\}$. When $\circ$ is present it is the largest element and thus a root. When a root is $\circ$, we don't draw it and simply draw and empty node in the picture. For $S \subset \mathbb{N}_{>0}$, we denote by $T \in \operatorname{Tree}_{S}$, the set of trees with set of nodes $S^{\circ}:=S \cup\{\circ\}$.

Example 2. We show below a forest $F$. The tree $F_{3}$ is a subtree of $F$, and $F_{8}$ is both a subtree and a tree of $F$, that is $F_{8} \in \operatorname{Tree}(F)$. We also examplify $F^{\dagger}$.

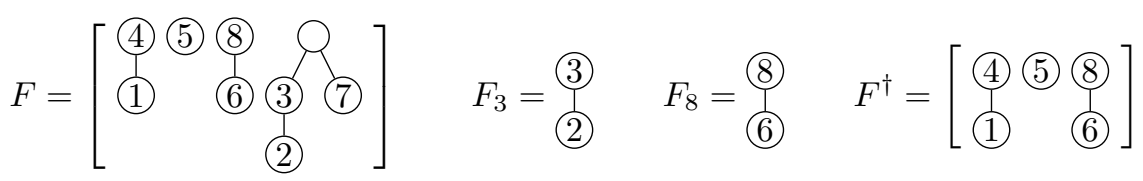

It is well known that the number of forest with set of node any given set of cardinality $k$ is $k$ ! (see. OEIS sequence A000142). A bijection is given in [HNT1, Section 3.2].

Definition 3. For any subset $S \subset \mathbb{N}$ we consider a family of vector fields $\left(\xi_{j}\right)_{j \in S}$ and a smooth section $A$ of the tensor bundle $\left(T_{X}^{*}\right)^{\otimes q} \otimes T_{X}^{\otimes h}$, with $q, h \geqslant 0$. For any tree $T$ with set of nodes contained in $S^{\circ}$ we define the nested derivative $\nabla_{\xi_{\bullet}}^{T} \xi_{\rho(T)}$ of $\xi_{\rho(T)}$, with $\xi_{\circ}:=A$ by the inductive formula

$$
\nabla_{\xi_{\bullet}}^{T} \xi_{\rho(T)}:=\left(\bigotimes_{\nu \in \operatorname{Child}(\rho(T))} \nabla_{\xi_{\bullet}}^{T_{\nu}} \xi_{\rho\left(T_{\nu}\right)}\right) \neg \nabla^{l_{\rho(T)}} \xi_{\rho(T)} .
$$


In particular if $S^{\circ}$ is the set of nodes of $T$, then

$$
\begin{aligned}
\nabla_{\xi_{\bullet}}^{T} A & =\left(\bigotimes_{\nu \in \operatorname{Child}(\rho(T))} \nabla_{\xi_{\bullet}}^{T_{\nu}} \xi_{\nu}\right) \neg \nabla^{l_{\rho(T)}} A, \\
\nabla_{\xi_{\bullet}}^{T_{\nu}} \xi_{\nu} & =\left(\bigotimes_{n \in \operatorname{Child}(\nu)} \nabla_{\xi_{\bullet}}^{T_{n}} \xi_{n}\right) \neg \nabla^{l_{\nu}} \xi_{\nu} .
\end{aligned}
$$

See Figure 2 for an example.

If we now apply recursively the chain rule

$$
\nabla_{\xi} \nabla_{\Xi_{1} \otimes \cdots \otimes \Xi_{k}}^{k} A=\nabla_{\xi \otimes \Xi_{1} \otimes \cdots \otimes \Xi_{k}}^{k+1} A+\sum_{j=1}^{k} \nabla_{\Xi_{1} \otimes \cdots \otimes \nabla_{\xi} \Xi_{j} \otimes \cdots \otimes \Xi_{k}}^{k} A
$$

to a tree, it writes as

$$
\nabla_{\xi_{j}} \nabla_{\xi_{\bullet}}^{T} A=\sum_{U} \nabla_{\xi_{\bullet}}^{U} A
$$

where the sum goes along the set of trees $U$ obtained by grafting $j$ to the left of any nodes of $T$. As a consequence, there are as many terms in this sum as nodes of $T$. We give here an example where a tree $T$ stands for $\nabla_{\xi_{\bullet}}^{T}$ :

$$
\nabla_{\xi_{1}}\left(\begin{array}{c}
P_{(6)} \\
(3) \\
(2)
\end{array}\right)=\left(\begin{array}{l}
1) \\
(2)
\end{array}\right.
$$

Note that if $T$ is strictly decreasing ordered and if $i$ is smaller than any nodes of $T$ then all the trees appearing in this sum are strictly decreasing ordered.

Applying iteratively this rule, since there is a unique way to get a strictly decreasing tree adding nodes one by one in the decreasing order, we get the multiple covariant derivative of $A$ :

$$
\left(\prod_{j \in S} \nabla_{\xi_{j}}\right) A=\sum_{T \in \operatorname{Tree}_{S}} \nabla_{\xi \bullet}^{T} A
$$

With respect to a Sobolev norm $\|\cdot\|_{r}$ we infer the inequality

$$
\left\|\left(\prod_{j \in S} \nabla_{\xi_{j}}\right) A\right\|_{r} \leqslant C_{r}^{|S|} \sum_{T \in \operatorname{Tree}_{S}}\left\|\nabla^{\ell(\rho(T))} A\right\|_{r} \cdot \prod_{\nu \in T^{*}}\left\|\nabla^{\ell(\nu)} \xi_{\nu}\right\|_{r} .
$$

We notice indeed the identity $|S|=\sum_{\nu \in T} \ell(\nu)$ for any $T \in$ Tree $_{S}$. 
Remark 2. The computation made there are very reminiscent to prelie computation. Indeed, it is well know that given a flat torsion-free connections and $\nabla$ its associated covariant derivative, the bilinear operator $X \triangleright Y:=\nabla_{X} Y$ endow the space of vector fields with a left pre-Lie algebra structure (see Man Proposition 3.1]). However in our case, the connection is not flat, so that we can't apply the pre-Lie calculus. Still, we can use Cayley trees by ensuring that when computing $\nabla_{X} Y$, the field $X$ is always a single leaf and not a proper tree.

\section{Multiple Lie derivatives of endomorphism sec- tions of the tangent bundle}

We notice now that for any $A$ smooth endomorphism section of $T_{X}$ and any torsion free connection $\nabla$ hold the identity

$$
L_{\xi} A=\nabla_{\xi} A+[A, \nabla \xi]
$$

We consider the operator ad $(A):=[A, \bullet]$ acting on endomorphism sections of $T_{X}$. Then the previous identity rewrites also as $L_{\xi}=\nabla_{\xi}-\operatorname{ad}(\nabla \xi)$ over the space of smooth endomorphism sections of $T_{X}$. In order to generalize this fomula to multiple Lie derivatives we need to introduce a few notations. We denote by $\mathcal{P}_{k}$ the set of partitions of the set $[k]$. We notice that for any $P \in \mathcal{P}_{k}$ there exists a unique $p \in P$ such that $\max p=k$. We denote $p_{k}$ such $p$. We denote by $P^{*}:=P \backslash\left\{p_{k}\right\}$. Moreover for any $p \in P$ we denote $p^{*}:=p \backslash\{\max p\}$. Given a family of smooth vector fields $\left(\xi_{j}\right)_{j=1}^{k}$ and a subset $S \subset[k]$ we denote by

$$
\nabla_{\xi \bullet}^{S}:=\prod_{j \in S} \nabla_{\xi_{j}},
$$

where the product is taken in the increasing order from the left to the right. This notation will only be used in this section.

Lemma 2. Let $\nabla$ be the extension to tensor sections of any torsion free connection. Then for any family of smooth vector fields $\left(\xi_{j}\right)_{j=1}^{k}$ the formula holds

$$
\prod_{j=1}^{k} L_{\xi_{j}}=\sum_{P \in \mathcal{P}_{k+1}}(-1)^{|P|-1}\left[\prod_{p \in P^{*}} \operatorname{ad}\left(\nabla_{\xi_{\bullet}}^{p^{*}} \nabla \xi_{\max p}\right)\right] \nabla_{\xi_{\bullet}}^{p_{k+1}^{*}}
$$

over the space of smooth endomorphism sections of $T_{X}$. The product on the right hand side is taken in the increasing order provided by the max of the elements of $P$, from the left to the right.

Before giving the proof, we provide an example of a summand in the righthand-side sum. We pick for $k=8$ the set partition

$$
P=\{\{2,3\},\{1,4,6\},\{7\},\{5,8,9\}\} .
$$

The associated summand is

$$
(-1)^{3} \operatorname{ad}\left(\nabla_{\xi_{2}} \nabla \xi_{3}\right) \operatorname{ad}\left(\nabla_{\xi_{1}} \nabla_{\xi_{4}} \nabla \xi_{6}\right) \operatorname{ad}\left(\nabla \xi_{7}\right) \nabla_{\xi_{5}} \nabla_{\xi_{8}} .
$$


Proof. We show the formula (4.1) by induction on $k$. By the inductive assumption

$$
\prod_{j=2}^{k+1} L_{\xi_{j}}=\sum_{P \in \mathcal{P}_{2, k+2}}(-1)^{|P|-1}\left[\prod_{p \in P^{*}} \operatorname{ad}\left(\nabla_{\xi_{\bullet}}^{p^{*}} \nabla \xi_{\max p}\right)\right] \nabla_{\xi_{\bullet}}^{p_{k+2}^{*}},
$$

where $\mathcal{P}_{2, k+2}$ denotes the set of partitions of the set $\{2, \ldots, k+2\}$. For any $P \in \mathcal{P}_{2, k+2}$ we denote by $\mathcal{P}_{P} \subset \mathcal{P}_{k+2}$ the subset of partitions obtained by adding 1 to one of the parts $p \in P$. We denote by $P_{1}:=\{\{1\}\} \cup P$. Then

$$
\begin{aligned}
\nabla_{\xi_{1}} \prod_{j=2}^{k+1} L_{\xi_{j}} & =\sum_{P \in \mathcal{P}_{2, k+2}}(-1)^{|P|-1} \nabla_{\xi_{1}}\left[\prod_{p \in P^{*}} \operatorname{ad}\left(\nabla_{\xi_{\bullet}}^{p^{*}} \nabla \xi_{\max p}\right)\right] \nabla_{\xi_{\bullet}}^{p_{k+2}^{*}} \\
& =\sum_{P \in \mathcal{P}_{2, k+2}}(-1)^{|P|-1} \sum_{P^{\prime} \in \mathcal{P}_{P}}\left[\prod_{p^{\prime} \in P^{\prime *}} \operatorname{ad}\left(\nabla_{\xi \bullet}^{p^{\prime *}} \nabla \xi_{\max p^{\prime}}\right)\right] \nabla_{\xi_{\bullet}}^{p_{k+2}^{\prime *}} \\
& =\sum_{P \in \mathcal{P}_{2, k+2}} \sum_{P^{\prime} \in \mathcal{P}_{P}}(-1)^{\left|P^{\prime}\right|-1}\left[\prod_{p^{\prime} \in P^{\prime *}} \operatorname{ad}\left(\nabla_{\xi_{\bullet}}^{p^{\prime *}} \nabla \xi_{\max p^{\prime}}\right)\right] \nabla_{\xi_{\bullet}}^{p_{k+2}^{* *}},
\end{aligned}
$$

and

$$
\begin{aligned}
& -\operatorname{ad}\left(\nabla \xi_{1}\right) \prod_{j=2}^{k+1} L_{\xi_{j}} \\
& =\sum_{P \in \mathcal{P}_{2, k+2}}(-1)^{|P|} \operatorname{ad}\left(\nabla \xi_{1}\right)\left[\prod_{p \in P^{*}} \operatorname{ad}\left(\nabla_{\xi_{\bullet}}^{p^{*}} \nabla \xi_{\max p}\right)\right] \nabla_{\xi_{\bullet}}^{p_{k+2}^{*}} \\
& =\sum_{P \in \mathcal{P}_{2, k+2}}(-1)^{\left|P_{1}\right|-1}\left[\prod_{p_{1} \in P_{1}^{*}} \operatorname{ad}\left(\nabla_{\xi_{\bullet}}^{p_{1}^{*}} \nabla \xi_{\max } p_{1}\right)\right] \nabla_{\xi_{\bullet}}^{p_{1, k+2}^{*}} .
\end{aligned}
$$

The conclusion

$$
\prod_{j=1}^{k+1} L_{\xi_{j}}=\sum_{P \in \mathcal{P}_{k+2}}(-1)^{|P|-1}\left[\prod_{p \in P^{*}} \operatorname{ad}\left(\nabla_{\xi_{\bullet}}^{p^{*}} \nabla \xi_{\max p}\right)\right] \nabla_{\xi_{\bullet}}^{p_{k+2}^{*}},
$$

follows from the fact that

$$
\mathcal{P}_{k+2}=\left[\bigsqcup_{P \in \mathcal{P}_{2, k+2}} \mathcal{P}_{P}\right] \bigsqcup\left[\bigsqcup_{P \in \mathcal{P}_{2, k+2}}\left\{P_{1}\right\}\right]
$$

Let $\mathcal{F}_{k^{\circ}}$ be the set of forest over $[k] \cup\{0\}$. Recall that, given a forest $F \in \mathcal{F}_{k^{\circ}}$ we denote $F_{\nu}$ the subtree rooted at $\nu$. We also denote by $l_{F}$ the number of trees 
of $F$. Recall also that $F^{\dagger}$ is the forest obtained by truncating from $F$ the tree $F_{\circ}$. Then Formula (4.1) combined with (3.3) implies

$$
\prod_{j=1}^{k} L_{\xi_{j}}=\sum_{F \in \mathcal{F}_{k^{\circ}}}(-1)^{l_{F}-1}\left[\prod_{T \in F^{\dagger}} \operatorname{ad}\left(\nabla_{\xi_{\bullet}}^{T} \nabla \xi_{\rho(T)}\right)\right] \nabla_{\xi_{\bullet}}^{F_{\bullet}},
$$

where the product is taken in the increasing order provided by the labels of the roots of $F^{\dagger}$, from the left to the right. Recall that for a tree $T$, we write $\nu \in T^{*}$ for $\nu \in T \backslash\{\rho(T)\}$. Then formula (4.2) and the inequality (3.4) imply the estimate

$$
\begin{aligned}
\left\|\left(\prod_{j=1}^{k} L_{\xi_{j}}\right) A\right\|_{r} & \leqslant C_{r}^{k} \sum_{F \in \mathcal{F}_{k^{\circ}}} 2^{l_{F}-1}\left(\left\|\nabla^{\ell(\circ)} A\right\|_{r} \cdot \prod_{\nu \in F_{\circ}^{*}}\left\|\nabla^{\ell(\nu)} \xi_{\nu}\right\|_{r}\right) \times \\
& \times \prod_{T \in F^{\dagger}}\left(\left\|\nabla^{1+\ell(\rho(T))} \xi_{\rho(T)}\right\|_{r} \cdot \prod_{\nu \in T^{*}}\left\|\nabla^{\ell(\nu)} \xi_{\nu}\right\|_{r}\right),
\end{aligned}
$$

where the power of two comes from the estimate of the ad operator.

\section{$5 \quad$ Forests and Dyck polynomials}

We define the monomial $X_{T}$ associated to a tree $T$ as

$$
X_{T}:=\prod_{\nu \in T} X_{\nu}^{\ell(\nu)+\delta_{\rho(T), \nu}},
$$

and the root-truncated monomial $X_{T}^{*}$ as

$$
X_{T}^{*}:=\prod_{\nu \in T^{*}} X_{\nu}^{\ell(\nu)} .
$$

Example 3. Considering the following tree

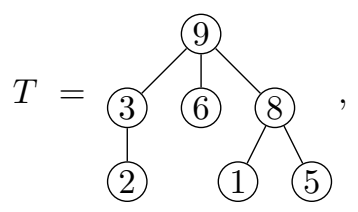

one finds $X_{T}=X_{1}^{0} X_{2}^{0} X_{3}^{1} X_{5}^{0} X_{6}^{0} X_{8}^{2} X_{9}^{4}$ and $X_{T}^{*}=X_{1}^{0} X_{2}^{0} X_{3}^{1} X_{5}^{0} X_{6}^{0} X_{8}^{2}$.

Let $B^{l}:=\left\|\nabla^{l} A\right\|_{r}$ and $X_{\nu}^{\ell(\nu)}:=\left\|\nabla^{\ell(\nu)} \xi_{\nu}\right\|_{r}$ in the estimate (4.3). The reader have to be careful that $X_{\nu}^{0}=\left\|\xi_{\nu}\right\| \neq 1$. Then the sum on the right hand side of (4.3) rewrites as

$$
\Sigma_{k}:=\sum_{F \in \mathcal{F}_{k^{\circ}}} 2^{l_{F}-1} B^{\ell_{F}(\circ)} X_{F_{\circ}}^{*} \prod_{T \in F^{\dagger}} X_{T} .
$$

With this notations we state the following theorem. 
Theorem 2. The sum $\Sigma_{k}$ satisfies the polynomial expression

$$
\Sigma_{k} \equiv \Sigma\left(X_{1}, \ldots, X_{k}\right)=\sum_{P \in \operatorname{Dyck}(k)} C_{P} B^{D_{P, k}} X_{1}^{p_{1}} \cdots X_{k}^{p_{k}} .
$$

Example 4. We give here the first few values of $\Sigma_{k}$.

$$
\begin{aligned}
\Sigma_{1} & =B X^{(0)}+2 X^{(1)}, \\
\Sigma_{2} & =B^{2} X^{(0,0)}+2 B X^{(1,0)}+3 B X^{(0,1)}+4 X^{(1,1)}+2 X^{(0,2)}, \\
\Sigma_{3} & =B^{3} X^{(0,0,0)}+2 B^{2} X^{(1,0,0)}+3 B^{2} X^{(0,2,0)}+4 B X^{(1,1,0)}+2 B X^{(0,1,0)} \\
& +4 B^{2} X^{(0,0,1)}+6 B X^{(1,0,1)}+9 B X^{(0,1,1)}+8 X^{(1,1,1)}+4 X^{(0,2,1)}+ \\
& +5 B X^{(0,0,2)}+4 X^{(1,0,2)}+6 X^{(0,1,2)}+2 X^{(0,0,3)} .
\end{aligned}
$$

From theorem 2 we infer the estimate

$$
\left\|\left(\prod_{j=1}^{k} L_{\xi_{j}}\right) A\right\|_{r} \leqslant C_{r}^{k} \sum_{P \in \operatorname{Dyck}(k)} C_{P}\left\|\nabla^{D_{P, k}} A\right\|_{r} \prod_{j=1}^{k}\left\|\nabla^{p_{j}} \xi_{j}\right\|_{r} .
$$

We need a few preliminaries in order to show theorem 2. Recall that a Dyck vector of length $k$ is a sequence $P \equiv\left(p_{1}, \ldots, p_{k}\right) \in \mathbb{N}^{k}$ such that

$$
\sum_{1 \leqslant r \leqslant j} p_{r} \leqslant j \quad \text { for all } j \in[k] .
$$

We denote their set by $\operatorname{Dyck}(k)$. A monomial $X^{P}=X_{1}^{p_{1}} \cdots X_{k}^{p_{k}}$ is Dyck monomial if $P$ is a Dyck vector.

Recall also that for any $F \in \mathcal{F}_{k}$ o we denote by $F_{\nu}$ the tree rooted at $\nu$ and by $F^{\dagger}$ the forest $F \backslash\left\{F_{\circ}\right\}$. For any forest $F \in \mathcal{F}_{k^{\circ}}$ we define

$$
X_{F}:=X_{F_{\circ}}^{*} \prod_{T \in F^{\dagger}} X_{T} .
$$

See Example 6 below. We observe that $X_{F}$ is always a Dyck monomial $X^{P}$. Indeed let

$$
p_{j}=\ell_{F}(j)+\delta_{j, \operatorname{Root}(F)} .
$$

for all $j=1, \ldots, k$ where $\delta_{j, \operatorname{Root}(F)}$ is 1 if $j$ is a root of $F$ and 0 otherwise. Then Condition (5.2) follows from the obvious identity

$$
\begin{aligned}
\sum_{1 \leqslant r \leqslant j} p_{r} & =\mid\{\nu \in F \cap[j] \mid \nu \text { child in } F \cap[j]\} \mid \\
& +\mid\{\nu \in F \cap[j] \mid \nu \text { root in } F\} \mid,
\end{aligned}
$$

and the fact that

$$
\{\nu \in F \cap[j] \mid \nu \text { child in } F \cap[j]\} \sqcup\{\nu \in F \cap[j] \mid \nu \text { root in } F\} \subseteq[j] .
$$

For any $P \in \operatorname{Dyck}(k)$, we define the set

$$
\mathcal{F}_{P}:=\left\{F \in \mathcal{F}_{k^{\circ}} \mid X_{F}=X^{P}\right\} .
$$


Example 5. We fix $P=(0,0,2,1,1)$. The following picture show all the forests such that $X_{F}=X^{P}$, sorted according to their length.
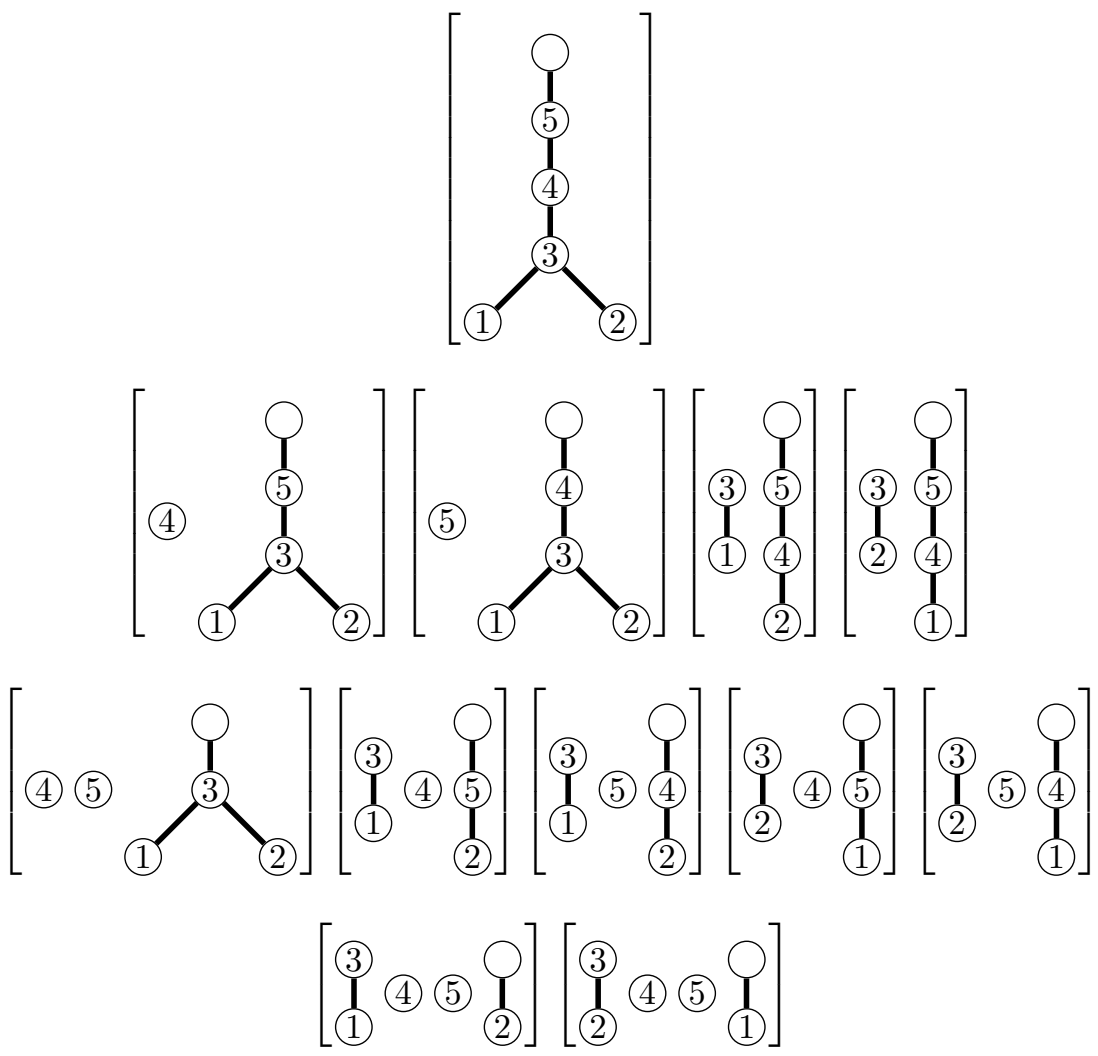

As a result we get that

$$
C_{(0,0,2,1,1)}=1+4 \cdot 2+5 \cdot 2^{2}+2 \cdot 2^{3}=45 .
$$

This agrees with

$$
\begin{aligned}
C_{(0,0,2,1,1)}=\left[2\left(\begin{array}{c}
0 \\
-1
\end{array}\right)+\right. & \left.\left(\begin{array}{l}
0 \\
0
\end{array}\right)\right]\left[2\left(\begin{array}{c}
1 \\
-1
\end{array}\right)+\left(\begin{array}{l}
1 \\
0
\end{array}\right)\right] \times \\
& \times\left[2\left(\begin{array}{l}
2 \\
1
\end{array}\right)+\left(\begin{array}{l}
2 \\
2
\end{array}\right)\right]\left[2\left(\begin{array}{l}
1 \\
0
\end{array}\right)+\left(\begin{array}{l}
1 \\
1
\end{array}\right)\right]\left[2\left(\begin{array}{l}
1 \\
0
\end{array}\right)+\left(\begin{array}{l}
1 \\
1
\end{array}\right)\right] .
\end{aligned}
$$

Definition 4. For any $F \in \mathcal{F}_{k^{\circ}}, k>0$ we denote by $F^{\prime} \in \mathcal{F}_{k}$ the forest obtained by pruning the childrens of the node labeled $\circ$ and grafting them, to the node labeled $k$. Of course, the old and new children of $k$ are shuffled to draw them in increasing order. Finally by replacing $k$ by $\circ$ in $F^{\prime}$, we consider that $F^{\prime}$ actually belongs to $\mathcal{F}_{k-1}$.

Example 6. It will becomes apparent in the following proofs that there are two different cases, whether $k$ is a child of $\circ$ or not. 
- We start by a case where $k=9$ is a not child of $\circ$ and thus a root of $F$. We show below a forest $F$ together with its associated $F^{\prime}$.

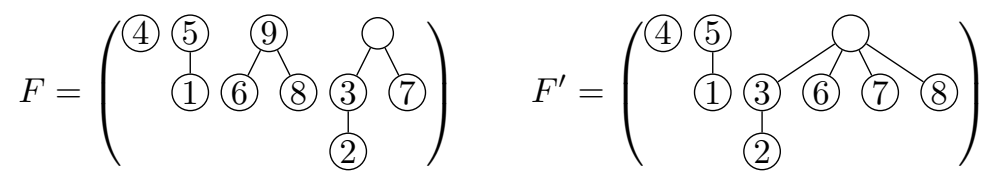

One can check that their associated monomials are $X_{F}=X^{(0,0,1,1,2,0,0,0,3)}$ and $X_{F^{\prime}}=X^{(0,0,1,1,2,0,0,0)}$.

- We show now a forest $F$ where $k=9$ is a child of $\circ$ together with its associated $F^{\prime}$.

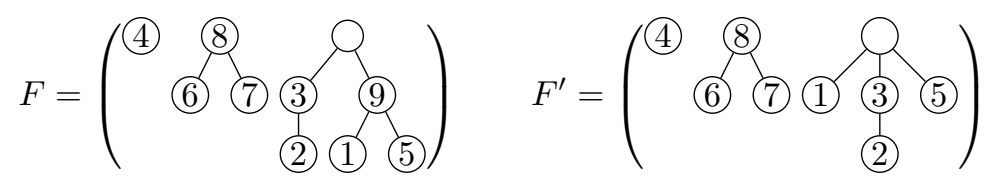

One can check that their associated monomials are $X_{F}=X^{(0,0,1,1,0,0,0,3,2)}$ and $X_{F^{\prime}}=X^{(0,0,1,1,0,0,0,3)}$.

Lemma 3. For any $F \in \mathcal{F}_{k^{\circ}}$, with $k \geqslant 0$ the identity $X_{F^{\prime}}=X_{F \mid X_{k}=1}$ holds.

Proof. By definition of $F^{\prime}$ we infer the identity $\ell_{F^{\prime}}(j)=\ell_{F}(j)$ for any node labeled $j<k$. Moreover the node labeled $j<k$ is a root in $F^{\prime}$ if and only if it is a root in $F$.

For $P=\left(p_{1}, \ldots, p_{k}\right)$, we denote by $\operatorname{deg}\left(X^{P}\right):=p_{1}+\cdots+p_{k}$.

Lemma 4. For any $F \in \mathcal{F}_{k^{\circ}}$, with $k \geqslant 0$ the identity $\ell_{F}(\circ)=k-\operatorname{deg}\left(X_{F}\right)$ holds. In particular $\ell_{F}(\circ)$ depends only on $X_{F}$ and not on $F$.

Proof. We prove this by induction on $k$. If $k=0$, there is only one forest $F \in \mathcal{F}_{\{\circ\}}$ whose monomial is $X_{F}=1$. We consider now a forest $F \in \mathcal{F}_{k^{\circ}}$ with $X_{F}=X_{1}^{p_{1}} \cdots X_{k}^{p_{k}}$. We reccal that the power $p_{j}$ of a monomial $X_{j}^{p_{j}}$ satisfies: $p_{j}=\ell_{F}(j)+1$ if $j=\rho(T)$ for some $T \in F$ and $p_{j}=\ell_{F}(j)$ if $j \neq \rho(T)$ for all $T \in F$. By the inductive assumption the statement hold for $F^{\prime}$. There are two case, whether $k$ is a child of $\circ$ or a root in $F$.

- If $k$ is not a child of o, i.e. a root, then $\ell_{F^{\prime}}(k)=\ell_{F}(k)+\ell_{F}(\circ)$. Therefore

$$
\begin{aligned}
\ell_{F}(\circ) & =\ell_{F^{\prime}}(k)-\ell_{F}(k) \\
& =k-1-\operatorname{deg}\left(X_{F^{\prime}}\right)-\left(p_{k}-1\right) \\
& =k-\operatorname{deg}\left(X_{F}\right) .
\end{aligned}
$$

The last equality follows from Lemma 3 
- If $k$ is a child of $\circ$, by definition of $F^{\prime}$, the childrens of $k$ in $F^{\prime}$ are the union of those of $k$ in $F$ and those of o exept $k$. Thus $\ell_{F^{\prime}}(k)=\ell_{F}(k)+\ell_{F}(\circ)-1$. We infer

$$
\begin{aligned}
\ell_{F}(\circ) & =1+\ell_{F^{\prime}}(k)-\ell_{F}(k) \\
& =1+\left(k-1-\operatorname{deg}\left(X_{F^{\prime}}\right)\right)-p_{k} \\
& =k-\operatorname{deg}\left(X_{F}\right) .
\end{aligned}
$$

The last equality follows from Lemma 3

Proof of theorem Q For any $P \in \operatorname{Dyck}(k)$, define

$$
C_{P}^{\prime}:=\sum_{F \in \mathcal{F}_{P}} 2^{l_{F}-1} .
$$

Then

$$
\Sigma_{k}=\sum_{F \in \mathcal{F}_{k}^{\circ}} 2^{l_{F}-1} B^{\ell_{F}(\circ)} X_{F}=\sum_{P \in \operatorname{Dyck}(k)} C_{P}^{\prime} B^{D_{P, k}} X^{P},
$$

thanks to lemma 4. Thus our goal is to show the equality

$$
C_{P}^{\prime}=\prod_{j=1}^{k}\left[2\left(\begin{array}{c}
D_{P, j-1} \\
p_{j}-1
\end{array}\right)+\left(\begin{array}{c}
D_{P, j-1} \\
p_{j}
\end{array}\right)\right]=C_{P} .
$$

We proceed by induction on $k$. We decompose $\mathcal{F}_{P}$ as a disjoint union as

$$
\mathcal{F}_{P}=\bigsqcup_{H \in \mathcal{F}_{p_{1}, \ldots p_{k-1}}} \mathcal{F}_{P}(H),
$$

where

$$
\mathcal{F}_{P}(H):=\left\{F \in \mathcal{F}_{P} \mid F^{\prime}=H\right\}
$$

As a consequence,

$$
C_{P}^{\prime}=\sum_{H \in \mathcal{F}_{p_{1}, \ldots, p_{k-1}}} \sum_{F \in \mathcal{F}_{P}(H)} 2^{l_{F}-1} .
$$

Thanks to Lemma 4, we infer $\ell_{H}(k)=k-1-\operatorname{deg}\left(X_{H}\right)$. By Lemma 3, we have $\operatorname{deg}\left(X_{H}\right)=p_{1}+\cdots+p_{k-1}$ so that

$$
\ell_{H}(k)=D_{P, k-1} .
$$

We now distinguish two cases, whether $k$ is a root of $F \in \mathcal{F}_{P}(H)$ or not: 
- The number of $F \in \mathcal{F}_{P}(H)$ such that $k$ is a root in $F$ is given by

$$
\left(\begin{array}{c}
D_{P, k-1} \\
p_{k}-1
\end{array}\right)
$$

Indeed for fixed $H$, the equality

$$
F \cap[k-1]=H \cap[k-1],
$$

shows that the only freedom of the forests $F \in \mathcal{F}_{P}(H)$ which satisfy (5.6) is in the choice of the the $\ell_{F}(k)=p_{k}-1$ childrens of $k$ in $F$ among the $\ell_{H}(k)=D_{P, k-1}$ childrens of $k$ in $H$. These childrens are given by the union of the childrens of $\circ$ and $k$ in $F$. The case $p_{k}=0$ does not occur since $k$ is a root in $F$. This is consistent with the convention $\left(\begin{array}{c}a \\ -1\end{array}\right)=0$.

Using the fact that, in this case, $l_{F}=l_{H}+1$, one conclude that for any fixed $H \in \mathcal{F}_{p_{1}, \ldots p_{k-1}}$, one has

$$
\sum_{\substack{F \in \mathcal{F}_{P}(H) \\
k \text { is a root of } F}} 2^{l_{F}-1}=2 \cdot 2^{l_{H}-1}\left(\begin{array}{c}
D_{P, k-1} \\
p_{k}-1
\end{array}\right) .
$$

- The number of $F \in \mathcal{F}_{P}(H)$ such that $k$ is not a root in $F$ is given by

$$
\left(\begin{array}{c}
D_{P, k-1} \\
p_{k}
\end{array}\right)
$$

The reason is the same as before. We notice that the definition of Dyck vector allows the case were $p_{k}=D_{P, k-1}+1$ (this is the case in Example1 for $k=5$ ). But $k$ has $D_{P, k-1}$ children in $F^{\prime}$ thanks to (5.5). Therefore it cannot have $p_{k}$ children in $F$. This is consistent with the convention $\left(\begin{array}{c}a \\ a+1\end{array}\right)=0$.

Using the fact that, in this case, $l_{F}=l_{H}$, one conclude that for any fixed $H \in \mathcal{F}_{p_{1}, \ldots p_{k-1}}$, one has

$$
\sum_{\substack{F \in \mathcal{F}_{P}(H) \\
k \text { is not a root of } F}} 2^{l_{F}-1}=2^{l_{H}-1}\left(\begin{array}{c}
D_{P, k-1} \\
p_{k}
\end{array}\right) .
$$

Combining Equation (5.4) with the two identities (5.7) and (5.8) we obtain

$$
\begin{aligned}
C_{P}^{\prime} & =\sum_{H \in \mathcal{F}_{p_{1}, \ldots p_{k-1}}}\left[2\left(\begin{array}{c}
D_{P, k-1} \\
p_{k}-1
\end{array}\right)+\left(\begin{array}{c}
D_{P, k-1} \\
p_{k}
\end{array}\right)\right] 2^{l_{H}-1} \\
& =C_{p_{1}, \ldots p_{k-1}}^{\prime}\left[2\left(\begin{array}{c}
D_{P, k-1} \\
p_{k}-1
\end{array}\right)+\left(\begin{array}{c}
D_{P, k-1} \\
p_{k}
\end{array}\right)\right] .
\end{aligned}
$$

We finally infer the required identity $C_{P}^{\prime}=C_{P}$ by induction on $k$. 
Remark 3. We notice the formula

$$
C_{P}=\prod_{\substack{1 \leqslant j \leqslant k \\
p_{j} \neq 0}}\left(2+\frac{D_{P, j}}{p_{j}}\right)\left(\begin{array}{c}
D_{P, j-1} \\
p_{j}-1
\end{array}\right) .
$$

Indeed

$$
C_{P}=\prod_{\substack{1 \leqslant j \leqslant k \\
p_{j} \neq 0}}\left[2\left(\begin{array}{c}
D_{P, j-1} \\
p_{j}-1
\end{array}\right)+\left(\begin{array}{c}
D_{P, j-1} \\
p_{j}
\end{array}\right)\right]
$$

and

$$
\left(\begin{array}{c}
D_{P, j-1} \\
p_{j}
\end{array}\right)=\frac{D_{P, j-1}-p_{j}+1}{p_{j}}\left(\begin{array}{c}
D_{P, j-1} \\
p_{j}-1
\end{array}\right) .
$$

Then the conclusion follows from the identity $D_{P, j}=D_{P, j-1}-p_{j}+1$.

\section{Higher order covariant derivatives of tensors}

In sequel, we denote for any $S \subset \mathbb{N}_{>0}$ we denote

$$
\nabla_{\xi \bullet}^{S}:=\left(\bigotimes_{p \in S} \xi_{p}\right) \neg \nabla^{|S|} .
$$

The reader should not confuse this with $\nabla_{\xi_{\bullet}}^{T}$ which is used when $T$ is a tree.

We set $\operatorname{Map}(h, l):=\{\mu:[h] \longrightarrow[l]\}$. Let $A_{j}$ be smooth sections of the bundle $\left(T_{X}^{*}\right)^{\otimes q_{j}} \otimes T_{X}, j=1, \ldots, l$. There are many situations in which the notion of product $\prod_{j=1}^{l} A_{j}$ is well defined. This is the case for instance when:

1. $q_{j}=1$ for all $j=1, \ldots, l-1$. In this case the product is just a composition $A_{1} \circ A_{2} \circ \cdots \circ A_{l}\left(\xi_{1} \otimes \cdots \otimes \xi_{q_{l}}\right)$ of endomorphisms with a $q_{l}$-linear map giving a $q_{l}$-linear map.

2. $q_{1}=l-1$ and $q_{j}=0,1$, for $j \geqslant 2$. In this second case, the product is the application of a $(l-1)$-linear map $A_{1}\left(A_{2}\left[\xi_{2}\right] \otimes A_{3}\left[\xi_{3}\right] \otimes \cdots \otimes A_{l}\left[\xi_{l}\right]\right)$ to either vector fields $A_{j}$ when $q_{j}=0$ or the value $A_{j}\left(\xi_{l}\right)$ of the linear map $A_{j}$ when $q_{j}=1$. The bracket around the $\left[\xi_{j}\right]$ means that they are only present if $q_{j}=1$. The result is a $q_{2}+q_{3}+\cdots+q_{l}$-linear map.

In all these cases the following lemma hold.

Lemma 5. Let $A_{j}$ be smooth sections of $\left(T_{X}^{*}\right)^{\otimes q_{j}} \otimes T_{X}, j=1, \ldots, l$ such that the formal product $\prod_{j=1}^{l} A_{j}$ is well defined and let $\left(\xi_{p}\right)_{p=1}^{h}$ be a family of vector fields over $X$. Then the $h$-order covariant derivative satisfies the general Leibnitz identity

$$
\left(\bigotimes_{p=1}^{h} \xi_{p}\right) \neg \nabla^{h}\left(\prod_{j=1}^{l} A_{j}\right)=\sum_{\mu \in \operatorname{Map}(h, l)}\left(\prod_{j=1}^{l} \nabla_{\xi_{\bullet}}^{\mu^{-1}(j)} A_{j}\right) .
$$


Proof. We proceed by induction. We remind first the inductive definition of higher order covariant derivative:

$$
\nabla_{\xi_{0} \otimes \cdots \otimes \xi_{h}}^{h+1}:=\nabla_{\xi_{0}} \nabla_{\xi_{1} \otimes \cdots \otimes \xi_{h}}^{h}-\sum_{p=1}^{h} \nabla_{\xi_{1} \otimes \cdots \otimes \nabla_{\xi_{0}} \xi_{p} \otimes \cdots \otimes \xi_{h}}^{h} .
$$

Taking a covariant derivative of the inductive assumption we infer

$$
\begin{aligned}
\nabla_{\xi_{0}}\left[\left(\bigotimes_{p=1}^{h} \xi_{p}\right) \neg \nabla^{h}\left(\prod_{j=1}^{l} A_{j}\right)\right] \\
\quad=\sum_{j=1}^{l}\left(\sum_{\mu \in \operatorname{Map}(h, l)} \nabla_{\xi_{\bullet}}^{\mu^{-1}(1)} A_{1} \cdots \nabla_{\xi_{0}} \nabla_{\xi_{\bullet}}^{\mu^{-1}(j)} A_{j} \cdots \nabla_{\xi_{\bullet}}^{\mu^{-1}(l)} A_{l}\right) .
\end{aligned}
$$

Thanks to the tensorial nature of the multi-covariant derivative, we can assume $\nabla_{\xi_{0}} \xi_{p}(x)=0, p=1, \ldots, k$ at some arbitrary point $x$. Then

$$
\begin{aligned}
\left(\bigotimes_{p=0}^{h} \xi_{p}\right) & \neg \nabla^{h+1}\left(\prod_{j=1}^{l} A_{j}\right) \\
& =\sum_{j=1}^{l}\left(\sum_{\mu \in \operatorname{Map}(h, l)} \nabla_{\xi \bullet}^{\mu^{-1}(1)} A_{1} \cdots \nabla_{\xi \bullet}^{\{0\} \cup \mu^{-1}(j)} A_{j} \cdots \nabla_{\xi \bullet}^{\mu^{-1}(l)} A_{l}\right) .
\end{aligned}
$$

The conclusion follows from the observation that

$$
\operatorname{Map}(\{0, \ldots, h\}, l)=\left\{\mu_{j} \mid \mu \in \operatorname{Map}(h, l), j \in[l]\right\},
$$

where the map $\mu_{j}:\{0, \ldots, h\} \longrightarrow[l]$ is defined as by $\mu_{j}(0)=j$ and $\mu_{j}(i)=\mu(i)$ for $i \neq 0$.

Corollary 1. If $\xi$ is a vector field over $X$ then

$$
\xi^{\otimes h} \neg \frac{1}{h !} \nabla^{h}\left(\prod_{j=1}^{l} A_{j}\right)=\sum_{H \in \mathbb{N}^{l}(h)} \prod_{j=1}^{l}\left(\xi^{\otimes h_{j}} \neg \frac{1}{h_{j} !} \nabla^{h_{j}} A_{j}\right) .
$$

We infer the inequality with respect to the pointwise max norm on multilinear forms

$$
\frac{1}{h !}\left\|\nabla^{h}\left(\prod_{j=1}^{l} A_{j}\right)\right\| \leqslant \sum_{H \in \mathbb{N}^{l}(h)} \prod_{j=1}^{l} \frac{1}{h_{j} !}\left\|\nabla^{h_{j}} A_{j}\right\| .
$$

The previous pointwise inequality leads to the global estimate

$$
\frac{1}{h !}\left\|\nabla^{h}\left(\prod_{j=1}^{l} A_{j}\right)\right\|_{r} \leqslant C_{r}^{l-1} \sum_{H \in \mathbb{N}^{l}(h)} \prod_{j=1}^{l} \frac{1}{h_{j} !}\left\|\nabla^{h_{j}} A_{j}\right\|_{r} .
$$




\section{Proof of the theorem 1}

In this final section, we need to consider trees on the union of two families of vector fields $\eta_{\bullet}, \xi_{\bullet}$. This means that our label set for the trees will be a subset of two copies of $\mathbb{N}_{\geq 1}$ together with the usual empty root $\circ$. To distinguish the two copies, we write them $\mathbb{N}_{\geq 1}^{\prime}=\left\{1^{\prime}, 2^{\prime}, \ldots\right\}$ and $\mathbb{N}_{\geq 1}=\{1,2, \ldots\}$. Recall that the ordered sum $S+T$ of two totally ordered $\operatorname{sets}\left(S, \leq_{S}\right)$ and $\left(T, \leq_{T}\right)$ is the disjoint union $S+T:=S \sqcup T$ together with the order $\leq_{S+T}$ which keeps the relative order of the sets and such that all the elements of $S$ are smaller than the element of $T$. Formally,

$$
x \leq_{S+T} y \quad \Longleftrightarrow \quad\left\{\begin{array}{l}
x \leq_{S} y \text { if } x, y \in S \text { or } \\
x \leq_{T} y \text { if } x, y \in T \text { or } \\
x \in S \text { and } y \in T .
\end{array}\right.
$$

Definition 5. Let $S^{\prime} \subset \mathbb{N}_{\geq 1}^{\prime}$ and $S \subset \mathbb{N}_{\geq 1} \cup\{0\}$. Let $\mu \in \operatorname{Map}\left(S^{\prime}, S\right)$ and $F$ a forest on $S$. We denote $\mu \cup F$ the forest on $S^{\prime}+S$ with father function $f$ defined by $f\left(i^{\prime}\right)=\mu\left(i^{\prime}\right)$ for $i^{\prime} \in S^{\prime}$ and $f(i)=F(i)$ for $i \in S$.

We remark that the roots of $\mu \cup F$ are the same as the roots of $F$ so that if $F$ is actually a tree $T$ then $\mu \cup T$ is also a tree.

Example 7. Let $S=\{1,3,4,5,8,9$, ० $\}$ and $S^{\prime}=\left\{1^{\prime}, 2^{\prime}, 4^{\prime}, 6^{\prime}, 7^{\prime}, 9^{\prime}\right\}$. Consider

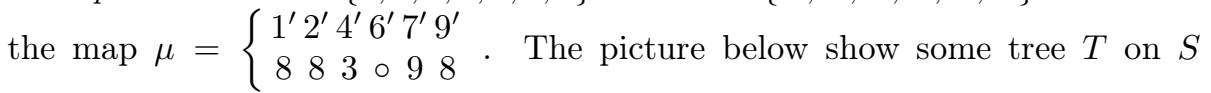
together with the associated $\mu \cup T$ tree:
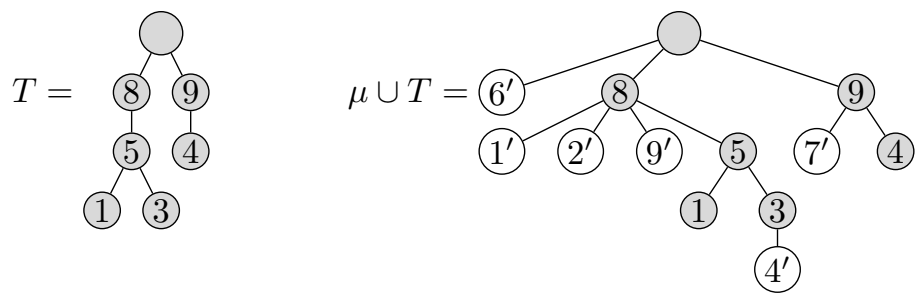

Expanding the associated nested derivative as in Definition 3 gives:

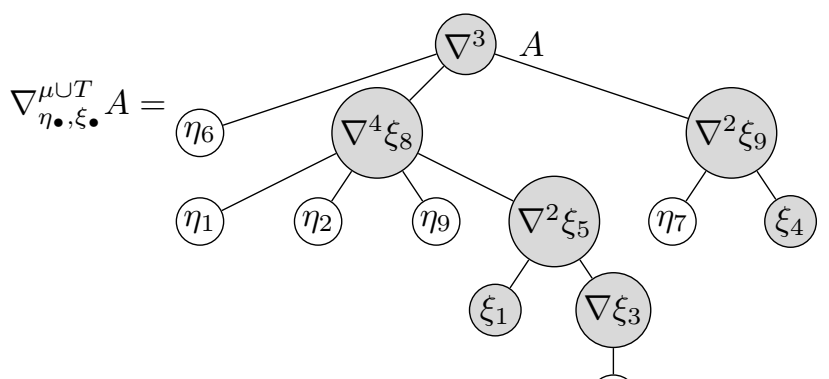

74 
We remark that for any node $\nu$ of $T$

$$
\ell_{\mu \cup T}(\nu)=\left|\mu^{-1}(\nu)\right|+\ell_{T}(\nu) .
$$

Moreover according to Definition $3, \nabla_{\eta_{\bullet}, \xi_{\bullet}}^{\mu \cup T} A$ is given by

$$
\nabla_{\eta_{\bullet}, \xi \bullet}^{\mu \cup T} A=\left[\left(\bigotimes_{p \in \mu^{-1}(\circ)} \eta_{p}\right) \otimes\left(\bigotimes_{\nu \in \operatorname{Child}(\circ)} \nabla_{\eta_{\bullet}, \xi_{\bullet}}^{\mu \cup T_{\nu}} \xi_{\nu}\right)\right] \neg \nabla^{\ell_{\mu \cup T}(\circ)} A
$$

with

$$
\nabla_{\eta_{\bullet}, \xi_{\bullet}}^{\mu \cup T_{\nu}} \xi_{\nu}=\left[\left(\bigotimes_{p \in \mu^{-1}(\nu)} \eta_{p}\right) \otimes\left(\bigotimes_{n \in \operatorname{Child}(\nu)} \nabla_{\eta_{\bullet}, \xi_{\bullet}}^{\mu \cup T_{n}} \xi_{n}\right)\right] \neg \nabla^{\ell_{\mu \cup T}(\nu)} \xi_{\nu},
$$

and so on, with $\nabla_{\eta_{\bullet}, \xi_{\bullet}}^{\mu \cup \emptyset}:=\mathbb{I}$ and the abuse of notation $\mu \cup H:=\left.\mu\right|_{\mu^{-1}(H)} \cup H$ for any subtree $H$ of $T$.

Proof of theorem [1. We apply recursively Lemma 5 in case 2 with

- $A_{1}:=\nabla^{l_{\rho(T)}} A$,

- $A_{i}:=\nabla_{\xi_{\bullet}}^{T_{\nu}} \xi_{\nu}$, if $i>1$, where $\nu$ is the $i$-th child of $\rho(T)$.

We get

$$
\nabla_{\eta_{\bullet}}^{S^{\prime}}\left(\nabla_{\xi \bullet}^{T} A\right) \equiv\left(\bigotimes_{p \in S^{\prime}} \eta_{p}\right) \neg \nabla^{\left|S^{\prime}\right|}\left(\nabla_{\xi_{\bullet}}^{T} A\right)=\sum_{\mu \in \operatorname{Map}\left(S^{\prime}, T\right)} \nabla_{\eta_{\bullet}, \xi_{\bullet}}^{\mu \cup T} A .
$$

By formula (4.2) and linearity, we get

$$
\begin{aligned}
& \left(\bigotimes_{p=1}^{h} \eta_{p}\right) \neg \nabla^{h}\left[\left(\prod_{j=1}^{k} L_{\xi_{j}}\right) A\right] \\
& \quad=\sum_{F \in \mathcal{F}_{k^{\circ}}}(-1)^{l_{F}-1}\left(\bigotimes_{p=1}^{h} \eta_{p}\right) \neg \nabla^{h}\left[\left(\prod_{T \in F^{\dagger}} \operatorname{ad}\left(\nabla_{\xi \bullet}^{T} \nabla \xi_{\rho(T)}\right)\right) \nabla_{\xi_{\bullet}}^{F_{\circ}} A\right] .
\end{aligned}
$$

We use now Lemma 5 in case 1 writing $F=\left\{T_{1}, \ldots, T_{l_{F}-1}, T_{l_{F}}=F_{\circ}\right\}$ in the increasing order provided by the labels of the roots of $F$, from the left to the right with

- $A_{i}:=\operatorname{ad}\left(\nabla_{\xi_{\bullet}}^{T_{i}} \nabla \xi_{\rho\left(T_{i}\right)}\right)$ for $i=1, \ldots, T_{l_{F}-1}$,

- $A_{l_{F}}:=\nabla_{\xi \bullet}^{F_{\circ}} A$. 
One obtains, for a fixed forest $F$,

$$
\begin{aligned}
G_{F} & :=\left(\bigotimes_{p=1}^{h} \eta_{p}\right) \neg \nabla^{h}\left[\left(\prod_{T \in F^{\dagger}} \operatorname{ad}\left(\nabla_{\xi \bullet}^{T} \nabla \xi_{\rho(T)}\right)\right) \nabla_{\xi_{\bullet}}^{F_{\circ}} A\right] \\
& =\sum_{\mu \in \operatorname{Map}\left(h, l_{F}\right)}\left[\prod_{j=1}^{l_{F}-1} \operatorname{ad}\left(\nabla_{\eta_{\bullet}}^{\mu^{-1}(j)} \nabla_{\xi_{\bullet}}^{T_{j}} \nabla \xi_{\rho\left(T_{j}\right)}\right)\right] \nabla_{\eta_{\bullet}}^{\mu^{-1}\left(l_{F}\right)} \nabla_{\xi_{\bullet}}^{F_{\circ}} A .
\end{aligned}
$$

Applying Equation 7.1 to each $T_{j}$, we obtain

$$
\begin{aligned}
G_{F} & =\sum_{\mu \in \operatorname{Map}\left(h, l_{F}\right)}\left[\prod_{j=1}^{l_{F}-1} \mathrm{ad}\left(\sum_{\beta_{j} \in \operatorname{Map}\left(\mu^{-1}(j), T_{j}\right)} \nabla_{\eta_{\bullet}, \xi_{\bullet}}^{\beta_{j} \cup T_{j}} \nabla \xi_{\rho\left(T_{j}\right)}\right)\right] \times \\
& \times\left(\sum_{\beta_{l_{F} \in \operatorname{Map}\left(\mu^{-1}\left(l_{F}\right), F_{\circ}\right)}} \nabla_{\eta_{\bullet}, \xi_{\bullet}}^{\beta_{l_{F} \cup F_{\circ}} A}\right) .
\end{aligned}
$$

We now recombine the maps $\mu,\left(\beta_{j}\right)_{j=1 . . l_{F}}$ into a single map $\alpha \in \operatorname{Map}\left(h, k^{\circ}\right)$ by setting $\alpha(p):=\beta_{\mu(p)}(p)$. Each $\alpha \in \operatorname{Map}\left(h, k^{\circ}\right)$ is obtained exactly once from a pair $\left(\mu,\left(\beta_{j}\right)_{j=1 . . l_{F}}\right)$. Then

$$
\begin{aligned}
\left(\bigotimes_{p=1}^{h} \eta_{p}\right) \neg \nabla^{h}\left[\left(\prod_{j=1}^{k} L_{\xi_{j}}\right) A\right] & \\
& =\sum_{\substack{F \in \mathcal{F}_{k^{\circ}} \\
\alpha \in \operatorname{Map}\left(h, k^{\circ}\right)}}(-1)^{l_{F}-1}\left[\prod_{T \in F^{\dagger}} \operatorname{ad}\left(\nabla_{\eta_{\bullet}, \xi \bullet \bullet}^{\alpha \cup T} \nabla \xi_{\rho(T)}\right)\right] \nabla_{\eta_{\bullet}, \xi \bullet}^{\alpha \cup F_{\bullet}} A,
\end{aligned}
$$

where we again used the abuse of notation $\alpha \cup T:=\left.\alpha\right|_{\alpha^{-1}(T)} \cup T$ for any subtree $T$ of $F$.

In the case $\eta=\eta_{p}$ for all $p$, we obtain as for the inequality (6.2)

$$
\begin{aligned}
& \frac{1}{h !}\left\|\nabla^{h}\left(\prod_{j=1}^{k} L_{\xi_{j}}\right) A\right\|_{r} \\
& \leqslant C_{r}^{k} \sum_{\substack{F \in \mathcal{F}_{k \circ} \\
H \in \mathbb{N}^{k+1}(h)}} 2^{l_{F}-1} \times \\
& \times\left(\frac{1}{h_{k+1} !}\left\|\nabla^{h_{k+1}+\ell(\circ)} A\right\|_{r} \cdot \prod_{\nu \in F_{\circ}^{*}} \frac{1}{h_{\nu} !}\left\|\nabla^{h_{\nu}+\ell(\nu)} \xi_{\nu}\right\|_{r}\right) \times \\
& \times \prod_{T \in F^{\dagger}}\left(\frac{1}{h_{\rho(T)} !}\left\|\nabla^{h_{\rho(T)}+1+\ell(\rho(T))} \xi_{\rho(T)}\right\|_{r} \cdot \prod_{\nu \in T^{*}} \frac{1}{h_{\nu} !}\left\|\nabla^{h_{\nu}+\ell(\nu)} \xi_{\nu}\right\|_{r}\right) .
\end{aligned}
$$


For any fixed $H \in \mathbb{N}^{k+1}(h)$ we consider the terms of the sum in (7.2) and we set $B^{l}:=\frac{1}{h_{k+1} !}\left\|\nabla^{h_{k+1}+l} A\right\|_{r}$ and $X_{\nu}^{l}:=\frac{1}{h_{\nu} !}\left\|\nabla^{h_{\nu}+l} \xi_{\nu}\right\|_{r}$. Then the estimate in the statement of theorem 1 follows from theorem 2

\section{Aknowledgment}

This research was driven by computer exploration, using the open-source mathematical software Sagemath Sage and its algebraic combinatorics features developed by the Sage-Combinat community [SC].

\section{References}

[Cay] Cayley, A., On the theory of the analytical forms called trees, Phil. Mag., 13 (1857), 172-6.

[HNT1] Hivert, F., Novelli, J.-C. And Thibon, J.-Y., Multivariate generalizations of the Foata-Schützenberger equidistribution, DMTCS Proceedings, AG, (2006), 289-300.

[HNT2] Hivert, F., Novelli, J.-C. And Thibon, J.-Y., Commutative combinatorial Hopf algebras, J. Algebr Comb, 28 , (2008) 65-95.

[Man] Manchon, D., A short survey on pre-Lie algebras, E. Schrödinger Institut Lectures in Math. Phys., Eur. Math. Soc, A. Carey Ed. (2011).

[Pal1] Pali, N., The Soliton-Kähler-Ricci Flow over Fano Manifolds, New York J. Math. 20, (2014), 845-919.

[Pal2] Pali, N., Variational stability of Kähler-Ricci solitons, Advances in Mathematics 290, (2016), 15-35.

[Pal3] Pali, N., The Soliton-Ricci Flow vith variable volume forms, Complex Manifolds, Vol 3, Issue 1, (2016), 41-144.

[P-S] Phong, D.H., Sturm, J., On stability and the convergence of the Kähler-Ricci flow, arXiv:0412185v1, (2004), J. Differential Geom. 72 (2006), no. 1, 149-168.

[Sage] Stein, W et Al., Sage Mathematics Software, The Sage Development Team. 2018 http://www. sagemath.org.

[SC] The Sage-Combinat community, Sage-Combinat: enhancing Sage as a toolbox for computer exploration in algebraic combinatorics, 2008, http://combinat.sagemath.org.

[OEIS] Sloane, N. J. A., The On-Line Encyclopedia of Integer Sequences, http://oeis.org. 
Florent Hivert

Laboratoire de Recherche en Informatique, Université Paris Sud,

Bâtiment 650 F91405 Orsay, France

E-mail: florent.hivert@lri.fr

Nefton Pali

Université Paris Sud, Département de Mathématiques

Bâtiment 307 F91405 Orsay, France

E-mail: nefton.pali@math.u-psud.fr 